\title{
Brain Circuit for Cognitive Control Is Shared by Task and Language Switching
}

\author{
Wouter De Baene ${ }^{1,2,3}$, Wouter Duyck ${ }^{1}$, Marcel Brass $^{1}$, and Manuel Carreiras ${ }^{2,4}$
}

\begin{abstract}
Controlling multiple languages during speech production is believed to rely on functional mechanisms that are (at least partly) shared with domain-general cognitive control in early, highly proficient bilinguals. Recent neuroimaging results have indeed suggested a certain degree of neural overlap between language control and nonverbal cognitive control in bilinguals. However, this evidence is only indirect. Direct evidence for neural overlap between language control and nonverbal cognitive control can only be provided if two prerequisites are met: Language control and nonverbal cognitive control should be
\end{abstract}

\section{INTRODUCTION}

A key question in bilingual language production is how bilingual speakers are able to control their two languages during speech processing and why they are so efficient in avoiding language conflicts or unintended nontarget language intrusions. The nature of the cognitive processes underlying this bilingual language control is still a matter of debate and has generated a substantial body of research during the last decade. Several language control mechanisms have been proposed, such as inhibition of the unintended language (Green, 1998).

One of the most frequently used paradigms to study the cognitive mechanisms underlying bilingual and multilingual language control in language production has been the language-switching paradigm (Abutalebi et al., 2008, 2013; Gollan \& Ferreira, 2009; Verhoef, Roelofs, \& Chwilla, 2009; Costa, Santesteban, \& Ivanova, 2006; Costa \& Santesteban, 2004; Jackson, Swainson, Cunnington, \& Jackson, 2001; Hernandez, Martinez, \& Kohnert, 2000; Meuter \& Allport, 1999). Recent neuroimaging research has suggested that brain areas involved in language switching are similar to those implicated in nonverbal cognitive control, as measured for instance with (nonverbal) taskswitching paradigms (e.g., Garbin et al., 2010, 2011; Guo, Liu, Misra, \& Kroll, 2011; Abutalebi \& Green, 2008; Wang, Xue, Chen, Xue, \& Dong, 2007; Crinion et al., 2006;

\footnotetext{
${ }^{1}$ Ghent University, ${ }^{2}$ Basque Center on Cognition, Brain and Language, Donostia-San Sebastián, Spain, ${ }^{3}$ Tilburg University,

${ }^{4}$ Basque Foundation for Science, Bilbao, Spain
}

compared within the same participants, and the task requirements of both conditions should be closely matched. To provide such direct evidence for the first time, we used fMRI to examine the overlap in brain activation between switch-specific activity in a linguistic switching task and a closely matched nonlinguistic switching task, within participants, in early, highly proficient Spanish-Basque bilinguals. The current findings provide direct evidence that, in these bilinguals, highly similar brain circuits are involved in language control and domaingeneral cognitive control.
Hernandez, Dapretto, Mazziotta, \& Bookheimer, 2001; Hernandez et al., 2000). The language control network involves lateral and medial prefrontal areas, parietal areas, and the caudate nucleus (see Abutalebi \& Green, 2008, for a review). On the other hand, in task switching, a frontoparietal network is generally observed, including lateral and medial prefrontal, premotor, and anterior and posterior parietal regions as well as the BG (De Baene, Albers, \& Brass, 2012; De Baene \& Brass, 2011; Shi, Zhou, Müller, \& Schubert, 2010; Crone, Wendelken, Donohue, \& Bunge, 2006; Yeung, Nystrom, Aronson, \& Cohen, 2006; Barber \& Carter, 2005; Ruge et al., 2005; Braver, Reynolds, \& Donaldson, 2003; Brass \& von Cramon, 2002; Dreher \& Berman, 2002; Dreher, Koechlin, Ali, \& Grafman, 2002; Rushworth, Hadland, Paus, \& Sipila, 2002; Rushworth, Paus, \& Sipila, 2001; Dove, Pollmann, Schubert, Wiggins, \& Yves von Cramon, 2000; Kimberg, Aguirre, \& D'Esposito, 2000; Sohn, Ursu, Anderson, Stenger, \& Carter, 2000).

Interestingly, up to now there is only indirect evidence that the neural regions supporting language control are the same as those supporting cognitive control in nonverbal domains. Only a few studies have directly examined the neural regions involved in a nonverbal cognitive control task in bilinguals (Garbin et al., 2010; Luk, Anderson, Craik, Grady, \& Bialystok, 2010; Bialystok et al., 2005). Garbin et al. (2010), for instance, compared the brain regions involved in a non-linguistic switching task between bilinguals and monolinguals and reported fundamental differences in the brain network engaged in task switching between both groups. Whereas monolinguals activated the right inferior frontal gyrus, ACC, and left inferior parietal 
lobule, bilinguals only displayed switch-specific activity in the left inferior frontal gyrus and the left striatum. Given that the left inferior frontal gyrus and the left striatum have been consistently related to bilingual language control (e.g., Abutalebi \& Green, 2007), according to Garbin et al. (2010), these results suggest a certain degree of neural overlap between language control and nonverbal cognitive control in bilinguals.

However, to directly examine the link between the regions involved in control of language conflict and those involved in general cognitive control, we need to examine the regions involved in both domains, within the same participants. Until now, only Abutalebi et al. (2012) followed this rationale. They examined within the same participants whether language control and the cognitive control processes involved in the flanker task have a common neural substrate. The dorsal ACC was found to be common to language switching and conflict monitoring in the flanker task. However, comparing a language-switching task with a conflict task confounds a number of cognitive control processes that are not related to the specific requirements of language switching. In particular, language switching relies mainly on the executive function of mental shifting (Miyake et al., 2000), whereas the flanker task is more strongly associated with inhibition of distractors or responses. Although these executive functions (together with updating of working memory) are moderately correlated (see also Friedman et al., 2006), they are clearly distinct, separable functions. Accordingly, these functions seem to rely on a partly shared-partly selective neural circuit. There are several brain areas involved commonly in different executive processes, whereas other brain areas are involved only in specific executive processes (e.g., only for shifting; Hedden \& Gabrieli, 2010). To capture those specific processes that relate language control with nonverbal control, one needs to compare language switching with a closely matched nonlinguistic switching paradigm. Therefore, we examined the neural overlap between two closely matched linguistic and nonlinguistic switching paradigms within the same participants.

Importantly, the occurrence and manifestation of language conflict might depend on the proficiency of the bilinguals (Van Heuven, Schriefers, Dijkstra, \& Hagoort, 2008). In fact, the precise nature of the language conflict (Abutalebi \& Green, 2007) and the associated control mechanism (Costa et al., 2006; Costa \& Santesteban, 2004) might even alter qualitatively with proficiency. At the neural level, the activation of the regions involved in language control or the specific network involved might also be modulated by language proficiency (Abutalebi et al., 2013; Garbin et al., 2011). Consequently, one might assume that also the overlap between the regions involved in language control and the regions involved in cognitive control might vary as a function of language proficiency. In this study, we only considered early proficient bilinguals who switch frequently between languages.
This choice was motivated by the fact that several studies have claimed that bilinguals outperform monolinguals on a range of cognitive control tasks (e.g., Prior \& Gollan, 2011; Prior \& MacWhinney, 2010; Bialystok \& Viswanathan, 2009; Bialystok, Craik, \& Luk, 2008; Costa, Hernández, \& Sebastián-Gallés, 2008; but see Antón et al., 2014; Duñabeitia et al., 2014; Hernández, Martin, Barceló, \& Costa, 2013; Paap \& Greenberg, 2013). However, this bilingual advantage might be more salient and might spread across a wider range of attention-demanding tasks (Bialystok, Craik, \& Ryan, 2006) for those bilinguals who constantly exercise language control functions on a daily basis (Verreyt, Woumans, Vandelanotte, Szmalec, \& Duyck, 2015).

In summary, in the current study, we wanted to examine the overlap in brain activation between a languageswitching paradigm and a nonverbal task-switching paradigm with a closely matched procedure, using a within-subject paradigm with early proficient bilinguals. This approach allows us to directly relate brain activation in a linguistic switching task to brain activation in a nonlinguistic switching task, providing the strongest test possible of the generalizability of the language control system developed by early proficient bilinguals to the cognitive control domain.

In this study, we opted for a paradigm with three tasks or three languages. This was motivated by the fact that two-task or two-language experiments might be a special case, because switching away from one task or language automatically involves switching back to the only other task or language (Ruthruff, Remington, \& Johnston, 2001). With three tasks or languages, a switch requires that participants choose which of the remaining tasks or languages to perform, which might be more representative of natural language processing. Because early, proficient bilinguals seem to apply the same languageswitching mechanism not only to the most proficient languages but also to weaker (L3) languages (Costa et al., 2006; Costa \& Santesteban, 2004), including a weaker third language here should not imply qualitatively different language switches.

\section{METHODS}

\section{Participants}

Thirty-six healthy right-handed college students participated in this study for monetary reimbursement. Four participants were excluded from the analyses because of excessive movement during scanning. All remaining participants $(13$ men; mean age $=22.4$ years, range $=$ 18-33 years) had Spanish as their L1, Basque as their L2 and had a good knowledge of English (L3). All participants were early, highly proficient bilinguals: They acquired L2 at an early age (on average before the age of 3 years; one participant at the age of 8 years) and were regularly (on average 4.3 days/week, range $=1-7$ days/week) 
confronted with contexts in which (inter- and intrasentential) code switching between their L1 and L2 occurred. The participants acquired L3 on average after the age of 6 years (range $=4-12$ years) and were rarely confronted with contexts in which code switching between their L1 and L3 or between their L2 and L3 occurred.

All participants had normal or corrected-to-normal vision. None of them used medication or had a history of drug abuse, head trauma, or neurological or psychiatric illness. All participants gave informed consent before testing. The study was approved by the institutional ethical committee.

\section{Materials}

Language proficiency has many different dimensions (word processing, syntactic processing, etc.), making it a complex concept to measure. Although, optimally, proficiency should be defined using different tasks that measure proficiency at different representational levels of the language, only single word processing tasks were included here given that the focus of this study is on switching at the word level. Next to the self-reported proficiency measures, language proficiencies in Spanish, Basque, and English were therefore measured with the Rapid Automatized Naming (RAN) test and the Boston Naming Test (BNT; see Table 1 for results on these tests).

\section{RAN Test}

Both a digits RAN test and a color RAN test were administered in Spanish, Basque, and English in all participants. The order of the language to be used was counterbalanced across participants. The RAN test (Denckla \& Rudel, 1974) is assumed to measure the ability to access and retrieve phonological representations from long-term memory (e.g., Torgesen, Wagner, Rashotte, Burgess, \& Hecht, 1997; Wagner \& Torgesen, 1987) as well as the ability to form orthographic representations (Bowers, Sunseth, \& Golden, 1999; Bowers, Golden, Kennedy, \& Young, 1994).

In each rapid naming test, participants were asked to name, as quickly as possible, six visual stimuli displayed on the screen, in a random order in four rows of nine stimuli each. Before each test, all stimuli were shown once to the participant to verify that he or she was able to name them in the languages to be used.

The stimuli for the digits RAN test were 1, 2, 3, 5, 7, and 8 , each presented six times. The stimuli of the color RAN test were red, black, green, brown, blue, and yellow squares, also presented six times each. Naming times were measured. The digits RAN test was always administered before the color RAN test.

As a proficiency measure, the ratio between the average naming times across the digits and color RAN tests in L2 and L1 was calculated. Perfectly balanced participants have a RAN ratio of 1 , whereas larger RAN ratios indicate a larger proficiency difference between L1 and L2.

\section{$B N T$}

The BNT was administered in Spanish, Basque, and English to all participants. The order of the language to be used was counterbalanced across participants. The BNT is assumed to measure word retrieval abilities (Kaplan, Goodglass, \& Weintraub, 1983).

The BNT contains 60 pictures presented one by one in order of word frequency and grade of difficulty (from common, high frequent, [e.g., "bed"] to less familiar, low frequent, [e.g., "abacus"]). Participants were asked to name them in the appropriate language. The scoring was done according to standard instructions.

\section{Language-switching Task}

For the language-switching task, eight pictures (size = $3.27 \times 3.27$ visual degrees) of common objects with noncognate names in Spanish, Basque, and English were selected from the Snodgrass and Vanderwart pictures set (Rossion \& Pourtois, 2004). The stimuli were selected based on the following matching criteria across the three languages: frequency, number of letters, number of phonemes, number of orthographic neighbors, age of acquisition, and concreteness. For Spanish and Basque, information was extracted from the BaSp database (Duñabeitia et al., in preparation). For English, information was provided by the N-Watch program (Davis, 2005).

Table 1. Overview of Language Proficiency Scores

\begin{tabular}{lcrr}
\hline & Spanish & Basque & English \\
\hline Self-ratings & $9.56(0.62)$ & $8.13(1.29)$ & $6.59(1.16)$ \\
Naming times (colors), sec & $21.59(2.75)$ & $25.65(4.22)$ & $26.70(6.61)$ \\
Naming times (numbers), sec & $14.04(2.79)$ & $17.30(4.80)$ & $19.06(2.95)$ \\
BNT & $50.72(3.79)$ & $39.97(8.71)$ & $24.72(7.41)$ \\
\hline
\end{tabular}


Figure 1. Design of the experiment. The languageswitching condition is presented on the left. The task-switching condition is presented on the right. A trial started with the presentation of a cue for $300 \mathrm{msec}$, which instructed the participants which language to use (Spanish, Basque, or English) or which task to perform (motion, color, or gender task). The cue was followed by the stimulus that was presented for $500 \mathrm{msec}$. The participants were instructed to respond as fast as possible,

without sacrificing accuracy. After the response (or maximally after $1500 \mathrm{msec}$, whichever came first), a variable response-cue interval started (mean = $2625 \mathrm{msec}$; range $=1000-5250 \mathrm{msec}$, in steps of $250 \mathrm{msec}$, distribution with pseudologarithmic density). In the language-switching condition, verbal responses were used whereas responses via button presses were used in the task-switching condition.

Participants were instructed to name the picture aloud in Spanish, Basque, or English according to the shape cue presented before the picture. Per participant, three cues were selected out of six available cues (a circle, diamond, triangle, square, star, or pentagon). The remaining three cues were used in the task-switching task. The cue-toresponse language assignments were counterbalanced across participants.

Each experimental trial had the following structure (Figure 1): After the presentation of a cue for $300 \mathrm{msec}$, a picture was presented on a black background at the center of the screen $(60 \mathrm{~Hz}$ frame rate, positioned $250 \mathrm{~cm}$ from the participants) for $500 \mathrm{msec}$, after which the participants had to respond as fast as possible, without sacrificing accuracy. After a jittered response-cue interval (mean $=2625 \mathrm{msec}$; range $=1000-5250 \mathrm{msec}$, in steps of $250 \mathrm{msec}$, distribution with pseudologarithmic density), the next trial started.

Before scanning, all participants completed a training phase. First, participants were familiarized with the names of the pictures in the three languages. To this end, each stimulus was presented centered on the screen with its name presented below it in Spanish, Basque, and English. Participants had to press a button to go to the next stimulus. After this familiarization phase, participants worked through one practice block for each language separately (16 trials each). The order of the language to be used in the practice blocks was counterbalanced across participants. Afterwards, participants worked through a practice block (48 trials) in which the three languages were randomly intermixed. In the scanner, participants went through nine blocks of 72 trials, each of which were equally distributed across the three languages and the eight stimuli. The sequence of trials was also controlled for an equal number of language transitions (e.g., L1-L1 vs. L1-L2) and language sequences (e.g., L1-L2-L1 vs. L3-L2-L1). Each block started with an instruction screen reminding the participants of the cue-to-language assignments. Speech onset of the vocal responses was recorded with a voice key. Errors were coded offline by the experimenter in a subject file.

\section{Task-switching Task}

In the task-switching task, three different tasks were used. In the motion task, participants judged the motion direction of the stimulus (up and down vs. left and right). In the color task, participants judged the color (red vs. blue) of the colored pixels of the stimulus. In the gender task, participants judged the gender (male vs. female) of the face. Participants used their index finger of their right and left hand to answer. The stimulus-response assignments for each task were counterbalanced across participants. On each trial, the task to perform was indicated by the shape cue presented before the stimulus. Per participant, three cues were selected out of six available cues (see previous section). The cue-to-task assignments were counterbalanced across participants.

All stimuli were stored as $320 \times 400$ pixel image sequences and presented for $500 \mathrm{msec}$ as a continuous movie of frame sequences at a frame rate of $60 \mathrm{~Hz}$ on a black background on a screen positioned $250 \mathrm{~cm}$ from the participant. The stimuli (size $=3.49 \times 4.36$ visual degrees) were pictures of a man or a woman filled with a random texture pattern (50\% colored and 50\% black pixels) moving at a standard speed of 1.3 degrees/sec. The colored pixels were either red or blue and were matched for luminance. The pixels moved up and down (250 msec each in intervals of $125 \mathrm{msec}$ ) or left and right (250 msec each in intervals of $125 \mathrm{msec}$ ). The structure of the experimental trials was identical to the languageswitching trial structure (Figure 1): After the presentation of a cue for $300 \mathrm{msec}$, a stimulus was presented for $500 \mathrm{msec}$ after which the participants had to respond as fast as possible, without sacrificing accuracy. After a jittered response-cue interval (mean $=2625 \mathrm{msec}$; 
range $=1000-5250 \mathrm{msec}$, in steps of $250 \mathrm{msec}$, distribution with pseudologarithmic density), the next trial started.

Before scanning, all participants went through a training phase. First, participants worked through one practice block for each task separately (16 trials each). The order of the tasks in the practice blocks was counterbalanced across participants. Afterwards, participants worked through a practice block ( 48 trials) in which the three tasks were randomly intermixed. In the scanner, participants went through nine blocks of 72 trials, each of which were equally distributed across the three tasks. The sequence of trials was also controlled for an equal number of task transitions (e.g., repeat vs. switch) and task sequences (e.g., color-motion-color vs. gender-motioncolor). Each block started with an instruction screen reminding the participants of the cue-to-task and stimulusresponse assignments.

\section{Procedure}

Given the amount of tasks and the duration of these tasks, participants went through two separate sessions, each lasting for about $2.5 \mathrm{hr}$, with a mean intersession time of 6.26 days ( $S D=2.78$ days).

One session contained the language-switching task. For half of the participants, the different RAN tests were also ran in this session, whereas the different BNT tests were ran in this session for the other half of the participants. The other session contained the task-switching task. Additionally, the different BNT tests were ran in this session for half of the participants, whereas the different RAN tests were added in the other half of the participants. The order of the two sessions was counterbalanced across participants.

\section{fMRI Data Acquisition and Analysis}

Participants were positioned head first and supine in the magnetic bore. Images were collected with a 3T Magnetom Trio MRI scanner system (Siemens Medical Systems, Erlangen, Germany), using a standard 32-channel radiofrequency head coil. Participants were instructed not to move their heads to avoid motion artifacts.

Each session started with a high-resolution 3-D structural scan, using a T1-weighted 3-D MPRAGE sequence (repetition time $=2530 \mathrm{msec}$, echo time $=2.97 \mathrm{msec}$, inversion time $=1100 \mathrm{msec}$, acquisition matrix $=256 \times$ $256 \times 176$, field of view $=256 \mathrm{~mm}$, flip angle $=7^{\circ}$, slice thickness $=1 \mathrm{~mm}$, slice gap $=0.5 \mathrm{~mm}$ ). Whole-brain functional images were collected using a T2*-weighted EPI sequence, sensitive to BOLD contrast (repetition time $=$ $2000 \mathrm{msec}$, echo time $=28 \mathrm{msec}$, image matrix $=64 \times 64$, field of view $=192 \mathrm{~mm}$, flip angle $=20^{\circ}$, slice thickness $=$ $3 \mathrm{~mm}$, distance factor $=20 \%$, voxels resized to $3 \times 3 \times$ $3 \mathrm{~mm}^{3}, 33$ axial slices). A varying number of images were acquired per run because of the self-paced initiation of trials.

\section{fMRI Data Preprocessing}

Data processing and analyses were performed using the SPM8 software (Wellcome Department of Cognitive Neurology, London, UK). The first four scans of all EPI series were excluded from the analysis to minimize T1 relaxation artifacts. Data processing started with slice time correction and realignment of the EPI datasets. A mean image for all EPI volumes was created, to which individual volumes were spatially realigned by rigid body transformation. The high-resolution structural image was coregistered with the mean image of the EPI series. The structural image was normalized to the Montreal Neurological Institute template. The normalization parameters were then applied to the EPI images to ensure an anatomically informed normalization. Motion parameters were estimated for each session separately. A commonly applied filter of 8-mm FWHM was used. The time series data at each voxel were processed using a high-pass filter with a cutoff of $128 \mathrm{sec}$ to remove low-frequency drifts.

Separately for the language-switching and task-switching parts, statistical analyses were performed on individual participants' data using the general linear model (GLM) in SPM8. The fMRI time series data were modeled by two different vectors reflecting the transition status (switch vs. repeat) of the trial. Erroneous trials and trials following errors were modeled together as a regressor of no interest and were excluded from the analyses.

All these vectors were convolved with the canonical hemodynamic response function, as well as with the temporal derivative and entered into the regression model (the design matrix). In addition, residual effects of head motion were corrected by including the six motion parameters estimated during the SPM8 realignment procedure for each participant as regressors of no interest in the design matrix. The statistical parameter estimates were computed separately for each voxel for all columns in the design matrix.

\section{Whole-brain Analyses}

For the group analyses, the contrast images from the single participant analyses were submitted to a randomeffects full factorial design with condition (language switching vs. task switching) as factor. Group map significance was defined using a threshold of $p<.0001$ at voxel level and a cluster level corrected for the whole brain at $p<.05$.

In a conjunction analysis, we compared the contrast images of both switching conditions to identify brain regions showing switch-specific activity common to both language switching and task switching. In this analysis, we tested for a rejection of the conjunction null hypothesis (i.e., only those voxels were reported as active which proved to be significant for the switch vs. repeat contrast in both switch conditions). Additionally, we used the contrast images of the language-switching and 
task-switching conditions for a disjunction analysis to identify areas showing switch-specific activity in language switching $(p<.0001)$ but not in task switching $(p>.10)$ as well as vice versa.

\section{ROI Analyses}

To get a more fine-grained look at the pattern of brain activation across conditions in the areas observed with the above-mentioned analyses, we performed an ROI analysis for each of these areas. Data for the different conditions (switch and repeat conditions for both the language-switching and task-switching parts) for each ROI were extracted from a 6-mm-radius sphere around the peak voxel identified for each of these areas.

For each ROI and participant, we also measured the voxelwise pattern of selectivity of the switch condition compared to the repeat condition. This was done by extracting a $t$ value for the contrast switch versus repeat at each voxel within the ROI (see also Peelen, Wiggett, \& Downing, 2006). A correlation between two switch selectivity patterns (i.e., in language switching and task switching) for each participant was calculated as follows. First, we computed a $t$ value for each voxel in the ROI reflecting language switch selectivity. Second, we computed a $t$ value for each voxel in the same ROI reflecting task switch selectivity. These two sets of $t$ values were then represented as two 1-D vectors. Finally, a correlation was computed between these two vectors. The average correlation across participants constitutes the voxelwise correlation between language switch and task switch selectivity. We would expect a positive voxelwise correlation between language switch selectivity and task switch selectivity in an ROI if the variation in selectivity across voxels is stable and reflects variations in the proportions of neurons exhibiting different kinds of selectivity (for a similar argument, see Peelen \& Downing, 2005). In summary, the assumption is that a positive correlation indicates that similar voxels are recruited during both switching conditions. As such, a positive voxelwise correlation within an area might provide additional evidence that this area is similarly involved in language control and in nonverbal cognitive control.

\section{RESULTS}

\section{Behavioral Results}

For the language production task, a GLM repeated-measures ANOVA was run on the accuracy data with Language (L1, L2, or L3) and Transition status (switch vs. repeat) as withinsubject variables. Only the main effect of Transition status reached significance, $F(1,31)=23.08, p<.001$. There was a switch cost with less accurate switch trials than language repeat trials (93.20\% vs. $96.06 \%$, respectively). This switch cost was equally large across languages (Interaction language $\times$ Transition status: $F<1$ ). Such a symmetric switch cost is generally found in balanced bilinguals, and switching between the early acquired languages also generalizes to a third, late acquired language (e.g., see previous findings in a different, Spanish-Catalan bilingual community; Costa et al., 2006). The main effect of Language was not significant, $F(2,30)=1.96, p=.16$. RT data for the language task were not available because the scanner noise yields technical difficulties for extracting the voice onset times.

For the task-switching task, a GLM repeated-measures ANOVA was run on both the accuracy and the RT data with Transition status (switch vs. repeat) as the withinsubject variable. A substantial switch cost was again observed: Participants were significantly less accurate (89.03\% vs. 93.39\%, $F(1,31)=29.37, p<.001)$ and slower $(808.1 \mathrm{msec}$ vs. $653.0 \mathrm{msec}, F(1,31)=119.19$, $p<.001)$ for switch trials than for repeat trials.

\section{fMRI Results}

\section{Conjunction Analysis}

We first tried to identify brain regions showing switchspecific activity common to both language switching and task switching. To do so, we ran a conjunction analysis of both the language-switching and task-switching conditions. This analysis (Figure 2; Table 2) revealed switch-specific activity in both conditions within the precuneus (extending into bilateral superior parietal lobule and left inferior parietal lobule), posterior cingulate cortex, left fusiform gyrus (extending into the cerebellum), pre-SMA, left inferior frontal junction (IFJ; extending into the inferior frontal gyrus), and left and medial calcerine fissure.

For each of these areas, an ROI analysis was performed to get a more fine-grained look at the pattern of brain activation across conditions. A GLM repeated-measures ANOVA for each of these areas with Activity as a dependent variable and Transition (switch vs. repeat) and condition (language switching vs. task switching) as independent variables showed a higher activity in switch trials than in repeat trials across conditions (main effect Transition, all $p s<.001)$ in all these areas. Additionally, the precuneus, posterior cingulate cortex, left IFJ, and pre-SMA also showed a significant interaction between Transition and Condition $(p<.05$ for posterior cingulate cortex; all other $p$ s $<.001$; for areas showing no interaction: all $p s>.36$ ). In these four areas, this interaction was driven by a higher switch-specific activity in task switching compared to language switching.

For each area, we also determined the voxel-by-voxel correlations between language switch selectivity and task switch selectivity. Voxel by voxel, language switch selectivity was significantly correlated with task switch selectivity in all ROIs: precuneus $\left(r=.46 ; t_{31}=6.03, p<.001\right)$, posterior cingulate cortex $\left(r=.61 ; t_{31}=11.04, p<.001\right)$, left fusiform gyrus $\left(r=.40 ; t_{31}=6.33, p<.001\right)$, pre-SMA 
Figure 2. Activation map for areas involved both in language switching and task switching averaged across 32 participants $(p<.0001$ uncorrected, corrected at cluster level) mapped onto a standard Colin brain template.
Table 2. Areas Common to Language Switching and Task Switching

\begin{tabular}{lccr}
\hline Area & Peak Coordinates & $z$ Score & Extent \\
\hline Precuneus & $-6-7652$ & 6.92 & 1041 \\
Posterior cingulum & $0-3431$ & 6.50 & 67 \\
Fusiform gyrus & $-45-67-17$ & 6.10 & 218 \\
Pre-SMA & 01452 & 6.06 & 211 \\
IFJ & -48831 & 5.99 & 267 \\
Calcarine fissure & $-12-7610$ & 4.61 & 40 \\
Calcarine fissure & $0-91-11$ & 4.54 & 69 \\
\hline
\end{tabular}

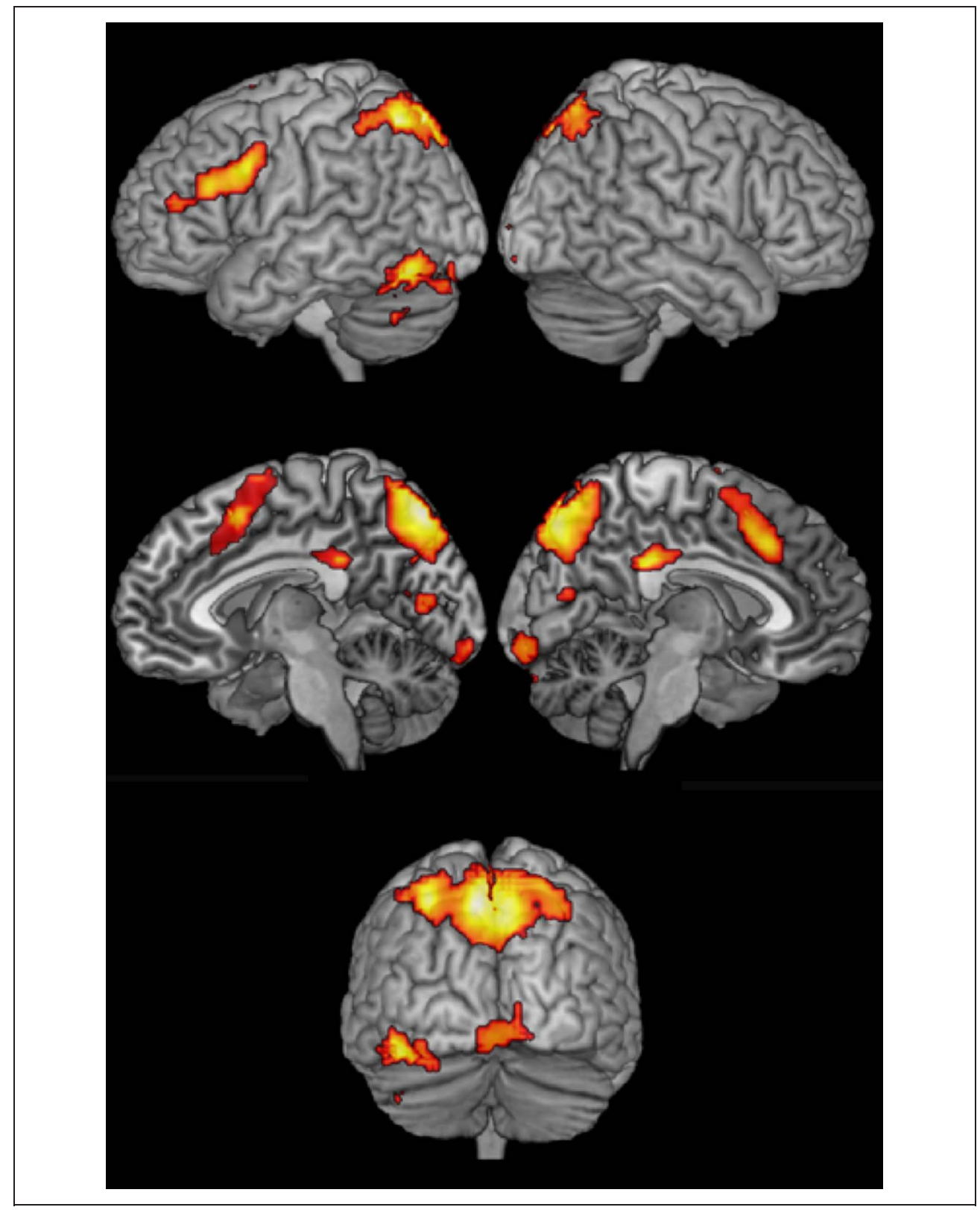

$\left(r=.56 ; t_{31}=7.54, p<.001\right)$, left IFJ $\left(r=.62 ; \mathrm{t}_{31}=10.79\right.$, $p<.001)$, left calcarine fissure $\left(r=.40 ; t_{31}=6.26, p<\right.$ $.001)$, and medial calcarine fissure $\left(r=.23 ; t_{31}=2.95\right.$, $p<.01)$. This suggests that in all these areas, similar subpopulations of neurons are recruited during both language switching and task switching.

\section{Disjunction Analyses}

To identify areas showing switch-specific activity specifically in language switching, we performed a disjunction analysis between language switching and task switching (Figure 3; Table 3). Switch-specific activity in right Sylvian fissure, pre-SMA, right precentral gyrus, and left precentral gyrus was only observed in language switching 
Figure 3. Activation map for areas specifically involved in language switching.

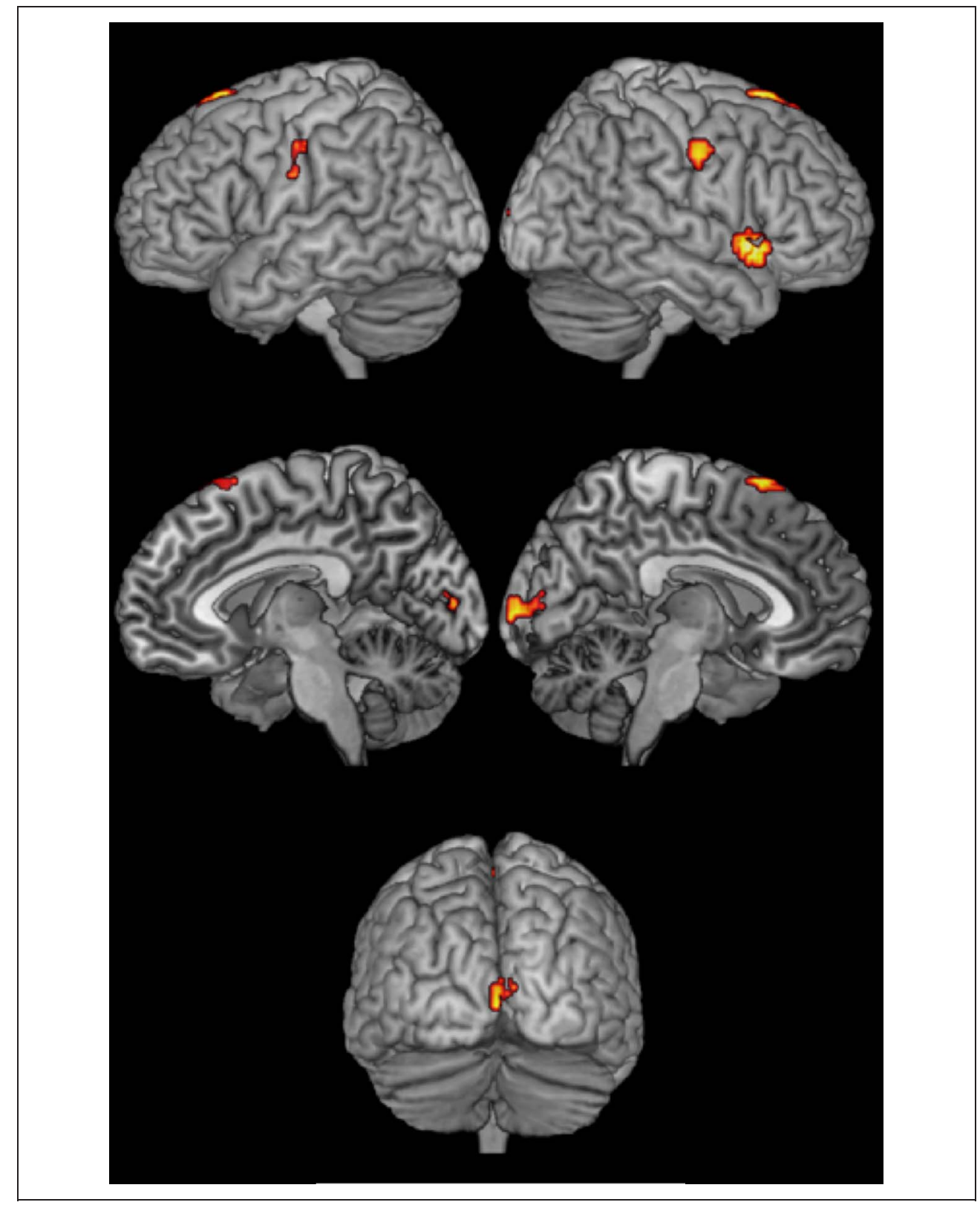

but not in task switching. To exclude the possibility that this result is merely the consequence of subthreshold activation in the task-switching condition in these areas and to provide additional support for the fact that these areas do show specific effects for language switching, ROI

Table 3. Areas Specifically Involved in Language Switching

\begin{tabular}{lccc}
\hline Area & Peak Coordinates & $z$ Score & Extent \\
\hline Sylvian fissure & $5417-5$ & 5.95 & 31 \\
Pre-SMA & 32067 & 5.23 & 27 \\
Precentral gyrus & $51-1040$ & 4.84 & 48 \\
Postcentral gyrus & $-45-1640$ & 4.57 & 38 \\
\hline
\end{tabular}

analyses in these areas were performed using a GLM repeated-measures ANOVA with Activity as a dependent variable and Transition (switch vs. repeat) and Condition (language switching vs. task switching) as independent variables. All areas showed a higher activity in switch trials compared with repeat trials across conditions (main effect Transition; $p<.05$ for left postcentral gyrus, $p<.01$ for right precentral gyrus, and $p<.001$ for right Sylvian fissure and pre-SMA). However, all these areas also showed a significant interaction between Condition and Transition (all $p s<.001)$. This interaction was driven by a significant language switch cost (all $p s<.001$ ) in combination with no task switch cost (all $p \mathrm{~s}>.15$ ).

To identify areas showing switch-specific activity specifically in task switching, we performed a disjunction analysis between task switching and language switching 
Figure 4. Activation map for areas specifically involved in task switching.

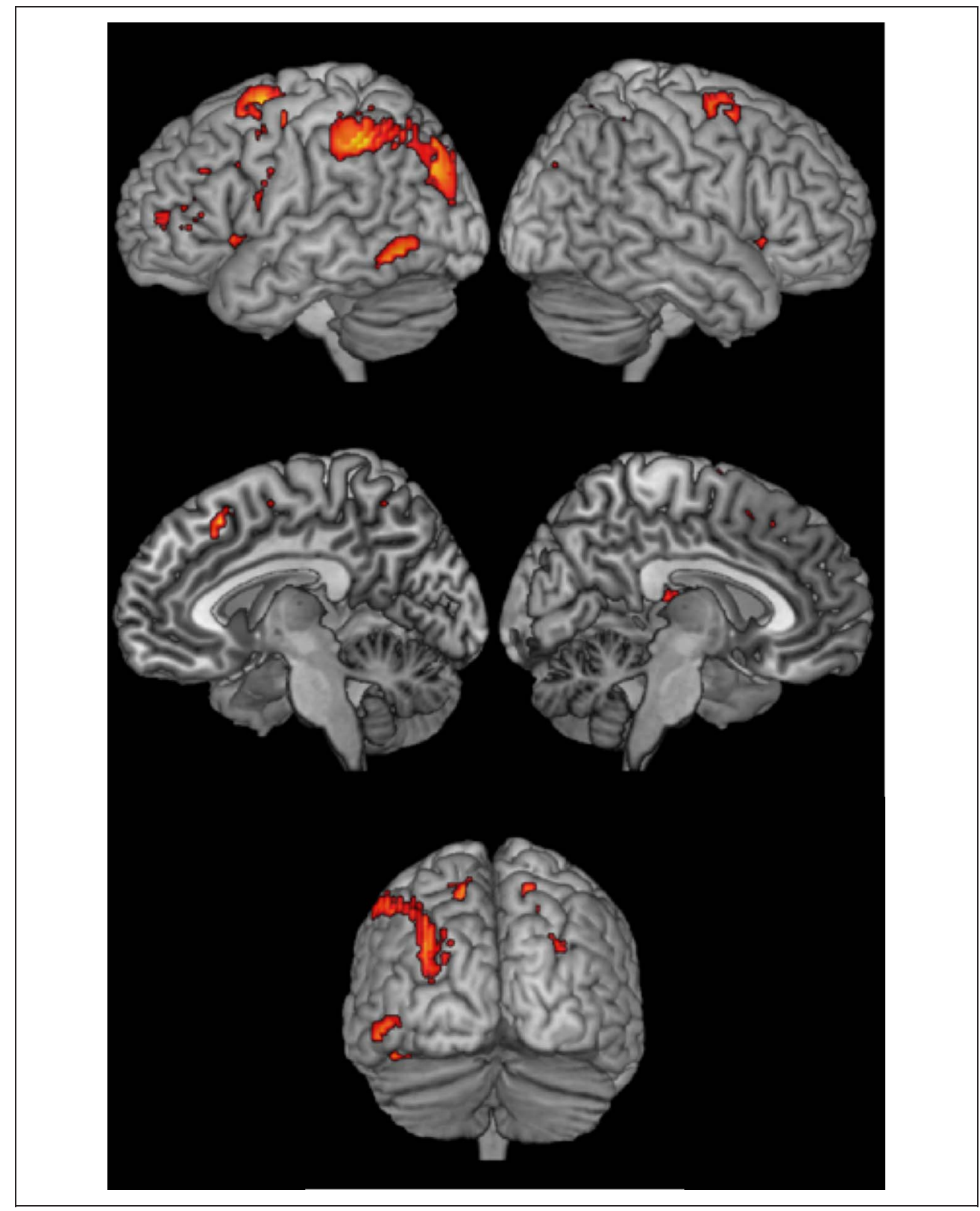

(Figure 4; Table 4). Switch-specific activity in left inferior parietal lobule, left superior frontal gyrus, right superior frontal sulcus, left middle occipital gyrus, right superior parietal lobule, right insula, and the rostral cingulate zone was only observed in task switching and not in language switching. To find additional support for these findings, ROI analyses in these areas using a GLM repeatedmeasures ANOVA with Activity as a dependent variable and Transition (switch vs. repeat) and Condition (language switching vs. task switching) as independent variables were performed. All areas showed a higher activity in switch compared to repeat trials across conditions (main effect Transition; all $p s<.001$ ). However, all these areas also showed a significant interaction between Condition and Transition (all $p s<.004$ ). For the left superior frontal gyrus, the right superior frontal sulcus, and the right
Table 4. Areas Specifically Involved in Task Switching

\begin{tabular}{lccr}
\hline Area & Peak Coordinates & $z$ Score & Extent \\
\hline Inferior parietal lobule & $-39-4046$ & 7.79 & 475 \\
Superior frontal gyrus & $-24-155$ & 7.73 & 486 \\
Superior frontal sulcus & $30-158$ & 6.28 & 85 \\
Middle occipital gyrus & $-48-58-11$ & 6.22 & 116 \\
Superior parietal lobule & $15-6155$ & 5.62 & 131 \\
Insula & 36177 & 4.98 & 33 \\
Rostral cingulate zone & 92049 & 4.94 & 30 \\
\hline
\end{tabular}


insula, this interaction is driven by a significant task switch cost (all $p s<.001$ ) in combination with no language switch cost (all $p$ s $>.32$ ). For left inferior parietal lobule and left middle occipital gyrus, this interaction is driven by a significant task switch cost (all $p s<.001$ ) in combination with a marginally significant language switch cost (all ps < .085). Finally, the interaction between condition and transition in the rostral cingulate zone and the right superior parietal lobule is driven by a combination of a significant task switch cost $(p<.001)$ with a significant but much smaller language switch cost (all $p$ s $<.05$ ).

\section{DISCUSSION}

Over the last decade, several studies have provided evidence that bilingual language control shares (at least partly) functional mechanisms with domain-general cognitive control (e.g., Calabria, Hernández, Branzi, \& Costa, 2012; Weissberger, Wierenga, Bondi, \& Gollan, 2012). Recently, attempts have been made to provide neural evidence for the bilingual overlap between language control and cognitive control (e.g., Abutalebi et al., 2012; Garbin et al., 2011; Abutalebi \& Green, 2007, 2008; Wang et al., 2007). The available neural evidence suggests that language control is achieved through multiple areas that are also engaged in cognitive control.

The support for this claim of neural overlap between language control and cognitive control in bilinguals, however, remained indirect. Direct evidence for neural overlap between two conditions may only be provided if both conditions are compared within the same participants. Furthermore, to capture the full scope of cognitive control processes involved in language switching, the task requirements of the different conditions need to be closely matched. Previous studies did not meet both prerequisites. The aim of the current study was to provide such direct evidence for the first time by examining the neural overlap between switch-specific activity in a linguistic switching task and a closely matched nonlinguistic switching task, within participants, in early, highly proficient bilinguals.

The current results support the claim that language control and more domain-general cognitive control in early, highly proficient bilinguals rely on common areas within the distributed frontoparietal network, which are also engaged in task-switching. Indeed, lateral and medial PFC as well as the inferior and superior parietal lobule were commonly active in linguistic and nonlinguistic switching. Furthermore, voxel-by-voxel analyses (e.g., Peelen et al., 2006) for all involved areas supported the similar contribution of these areas across linguistic and nonlinguistic switching. Consequently, the functions that are typically attributed to these areas for task switching could also apply for language switching.

Classically, the lateral PFC is linked to the maintenance, retrieval, and implementation of task goals and in performance adjustments by engaging regulatory processes to overcome interference and resolve competition from the previously implemented task set (e.g., Hyafil, Summerfield, \& Koechlin, 2009; MacDonald, Cohen, Stenger, \& Carter, 2000; Sohn et al., 2000). This fits the role proposed for lateral PFC in language switching in which the relevant language needs to be retrieved and implemented while resolving competition with the no-longer relevant language (see Abutalebi \& Green, 2007).

The medial PFC (comprising dorsal ACC and pre-SMA) has generally been attributed a monitoring and configuration role (e.g., Hyafil et al., 2009; Ridderinkhof, Ullsperger, Crone, \& Nieuwenhuis, 2004). The dACC detects conflict between, for instance, the previous and the new task in case of a task change (Ridderinkhof et al., 2004). The pre-SMA configures the cognitive system for the upcoming task by resolving the conflict by suppressing active but inappropriate actions from a previous task set and boosting the selection of appropriate actions as demanded by the new task set (Hikosaka \& Isoda, 2010; Isoda \& Hikosaka, 2007). Similarly, the medial PFC has been suggested to monitor the language context for bilingual or multilingual speakers (Abutalebi et al., 2013) and to withhold the language not in use (see Abutalebi \& Green, 2007). Additional evidence for the domain-general involvement of medial PFC in detecting and aiding the resolution of conflicts comes from a recent study of Abutalebi et al. (2012). They showed that the dACC and pre-SMA were the only areas that were common to a language control task and a flanker task in highly proficient bilinguals. Although the peak coordinates of the medial frontal area observed in the current study are slightly more anterior $(x=0, y=14, z=52$ vs. $x=0, y=2, z=60)$, this area overlaps with the pre-SMA reported by Abutalebi et al. (2012).

The superior parietal lobule has previously been shown to be involved in switching the attentional focus to the newly relevant task information when a change is detected (e.g., Braver et al., 2003). Furthermore, Mevorach, Humphreys, and Shalev (2006) showed that left and right posterior parietal cortex have complementary roles, respectively pulling attention away and pushing attention to the stimuli. Similarly, Abutalebi and Green $(2007,2008)$ proposed that also in unpredictable language switching, the left posterior parietal cortex might bias the attention away from the previous, now irrelevant language whereas the right part might bias the attention towards the new, relevant language.

Finally, the inferior parietal lobule (and sulcus) is commonly thought to be important for integration of sensory, cognitive, and motor information (Gottlieb, 2007; Andersen \& Buneo, 2002; Pouget, Deneve, \& Duhamel, 2002). These areas are assumed to be involved in representing and maintaining cue-associated response contingencies (Bunge, Kahn, Wallis, Miller, \& Wagner, 2003) or stimulus-response mappings (e.g., De Baene et al., 2012; Hartstra, Kühn, Verguts, \& Brass, 2011; Woolgar, 
Thompson, Bor, \& Duncan, 2011; Hester, D'Esposito, Cole, \& Garavan, 2007; Brass \& von Cramon, 2004). This is in line with the assumption that the inferior parietal lobule is related to the maintenance of word representations (Wang, Kuhl, Chen, \& Dong, 2009) in language switching.

Although early, highly proficient bilinguals seem to rely on common areas within the distributed frontoparietal network in language switching and task switching, some areas seem specifically involved in one of the two conditions, as is evident from the disjunction analyses. This might follow from the fact that the language-switching and task-switching paradigms, although matched to a very high degree, do necessarily differ in some respects. For instance, because we wanted to compare a pure linguistic task with a pure nonlinguistic task, the response modality is different in the two conditions: Whereas language switching is generally examined using verbal responses, button presses were used to respond in the task-switching condition. This could explain why switchspecific activation in precentral and postcentral gyri were only observed in language switching. These areas have been related to articulatory processing (Hillis et al., 2004) and are assumed to reflect the retrieval of stored phonological representations in overt naming (Murtha, Chertkow, Beauregard, \& Evans, 1999). All these processes are not involved in the nonlinguistic switching condition.

By contrast, the superior frontal gyrus and superior frontal sulcus, areas corresponding to the dorsal premotor cortex (Mayka, Corcos, Leurgans, \& Vaillancourt, 2006), were only observed in task switching. The dorsal premotor cortex integrates multiple sets of information on actions and integrates them to perform an intended action (Hoshi \& Tanji, 2007; O'Shea, Johansen-Berg, Trief, Göbel, \& Rushworth, 2007; Serrien, Ivry, \& Swinnen, 2007). As such, the dorsal premotor cortex executes the specific arbitrary association between a stimulus and a response in task switching (Badre \& D'Esposito, 2009). Whereas the association between a stimulus and the button response in task switching is indeed totally arbitrary, this is less so for the association between a picture and its name. This could explain why these areas were not observed in language switching. Alternatively, the observation of switch-specific activation in dorsal premotor cortex only in task switching could be explained by different switching demands in the language-switching and task-switching conditions. In a recent meta-analysis, Kim, Cilles, Johnson, and Gold (2012) showed that the dorsal premotor cortex is mainly involved in perceptual switching and does not contribute to switching between response mappings. Perceptual switching refers to switching attention between perceptual features of a stimulus. This is exactly what our participants needed to do in the taskswitching condition: They needed to switch their attention between perceptual features of the stimulus, namely the direction of motion of the moving noise, the color of the pixels and the gender of the face. This switching between perceptual stimulus features was not involved in language switching. Here, they needed to select and switch between different responses associated with the same stimulus.

The network of areas common to language switching and task switching observed here comprise all areas proposed by Abutalebi and Green (2007), except for the caudate nucleus. The role of the caudate in language switching remains puzzling as some studies report its activation (e.g., Abutalebi et al., 2008, 2013; Garbin et al., 2011; Wang et al., 2007; Crinion et al., 2006; for a meta-analysis, see Luk, Green, Abutalebi, \& Grady, 2012) whereas others do not (Hernandez, 2009; Wang et al., 2009; Hernandez et al., 2001). One possible interpretation for the absence of the caudate in the current study is that it is a consequence of the use of three different languages and the associated distribution of switch and repeat trials in the different languages. In a recent study, Ma et al. (2014) found the caudate when contrasting the switch condition with a simple naming condition in L1. However, the caudate was not observed when comparing the switch condition with a simple naming condition in L2. Ma et al. (2014) concluded that the caudate is involved in conditions that require much inhibition, hence in language switching and during the L2 naming condition, when inhibition of L1 is necessary. Consequently, the caudate should also be involved during L3 naming. Therefore, in the current study, the caudate might be involved in all switching conditions and in L2 and L3 repeat conditions. If the caudate is not activated in only one condition (i.e., the L1 repeat condition) out of six conditions in total, the contrast between switch and repeat conditions across languages might not be sensitive enough to capture this activation.

Note that some previous studies presented the language cue simultaneously with the stimulus (e.g., Abutalebi et al., 2008, 2013; Garbin et al., 2011; Guo et al., 2011) whereas others, including this study, presented the cue slightly before the stimulus (200-400 msec; e.g., Ma et al., 2014; Hernandez, 2009; Wang et al., 2007, 2009; Hernandez et al., 2000, 2001). We think that both approaches have advantages and disadvantages. The advantage of the simultaneous presentation of cue and target is to exclude task preparation and therefore amplifies switch costs. The disadvantage is that visual processing of the cue and cue-task translation takes place while the target is already presented. This can be ruled out by using a small cue-target interval (CTI) that warrants that participants can process the cue before the target appears. A CTI of $300 \mathrm{msec}$ does not leave much room for advance preparation because this time period is presumably necessary to visually process the cue and translate it into a task instruction. In any case, although manipulations of the CTI have substantial effects on performance, the influence on neural activity is restricted. Brass and von Cramon (2002), for example, showed that brain activity as measured with fMRI does not differ substantially for short and long CTIs. The reason is that participants need 
to establish the task-set regardless of the CTI. Because the BOLD response is not sensitive to small timing variations, delays in the preparation process of a few hundred milliseconds do not show up in the BOLD response. Therefore, we are convinced that this precuing has not markedly affected our results and does not hinder a direct comparison of the current results with previously reported findings of studies where no precuing has been used.

To conclude, the current findings provide direct evidence that in early, highly proficient bilinguals, highly similar brain circuits are involved in language control and domain-general cognitive control. Importantly, we have shown a more extensive overlap of regions for the two tasks than previously shown, given the direct contrast of language switching and task switching in the same highly proficient individuals.

\section{Acknowledgments}

This research was made possible by the Research FoundationFlanders (FWO-Vlaanderen; FWO10/PDO/234 and FWO13/ PDOH1/234), of which the first author is a postdoctoral research fellow, and further supported by the Special Research Fund (BOF) of Ghent University (BOF06/24JZAP and BOF08/GOA/ 011), from grant PSI2012-31448 from the Spanish Ministry of Science and Innovation and from grant ERC-2011-ADG-295362 from the European Research Council.

Reprint requests should be sent to Wouter De Baene, Department of Experimental Psychology, Ghent University, Henri Dunantlaan 2, Ghent, B-9000, Belgium, or via e-mail: Wouter.DeBaene@ ugent.be.

\section{REFERENCES}

Abutalebi, J., Annoni, J. M., Zimine, I., Pegna, A. J., Seghier, M. L., Lee-Jahnke, H., et al. (2008). Language control and lexical competition in bilinguals: An event-related fMRI study. Cerebral Cortex, 18, 1496-1505.

Abutalebi, J., Della Rosa, P. A., Ding, G., Weekes, B., Costa, A., \& Green, D. W. (2013). Language proficiency modulates the engagement of cognitive control areas in multilinguals. Cortex, 49, 905-911.

Abutalebi, J., Della Rosa, P. A., Green, D. W., Hernandez, M., Scifo, P., Keim, R., et al. (2012). Bilingualism tunes the anterior cingulate cortex for conflict monitoring. Cerebral Cortex, 22, 2076-2086.

Abutalebi, J., \& Green, D. (2007). Bilingual language production: The neurocognition of language representation and control.

Journal of Neurolinguistics, 20, 242-275.

Abutalebi, J., \& Green, D. W. (2008). Control mechanisms in bilingual language production: Neural evidence from language switching studies. Language and Cognitive Processes, 23, 557-582.

Andersen, R. A., \& Buneo, C. A. (2002). Intentional maps in posterior parietal cortex. Annual Review of Neuroscience, $25,189-220$.

Antón, E., Duñabeitia, J. A., Estévez, A., Hernández, J. A., Castillo, A., Fuentes, L. J., et al. (2014). Is there a bilingual advantage in the ANT task? Evidence from children. Frontiers in Psychology, 5, 398.

Badre, D., \& D'Esposito, M. (2009). Is the rostro-caudal axis of the frontal lobe hierarchical? Nature Reviews Neuroscience, 10, 659-669.
Barber, A. D., \& Carter, C. S. (2005). Cognitive control involved in overcoming prepotent response tendencies and switching between tasks. Cerebral Cortex, 15, 899-912.

Bialystok, E., Craik, F. I., Grady, C., Chau, W., Ishii, R., Gunji, A., et al. (2005). Effect of bilingualism on cognitive control in the Simon task: Evidence from MEG. Neuroimage, 24, 40-49.

Bialystok, E., Craik, F., \& Luk, G. (2008). Cognitive control and lexical access in younger and older bilinguals. Journal of Experimental Psychology: Learning, Memory, and Cognition, 34, 859.

Bialystok, E., Craik, F. I. M., \& Ryan, J. (2006). Executive control in a modified antisaccade task: Effect of aging and bilingualism. Journal of Experimental Psychology: Learning, Memory, and Cognition, 32, 1341-1354.

Bialystok, E., \& Viswanathan, M. (2009). Components of executive control with advantages for bilingual children in two cultures. Cognition, 112, 494-500.

Bowers, P., Golden, J., Kennedy, A., \& Young, A. (1994). Limits upon orthographic knowledge due to processes indexed by naming speed. In V. W. Berninger (Ed.), The varieties of orthographic knowledge (pp. 173-218). Dordrecht: Kluwer Academic Press.

Bowers, P. G., Sunseth, K., \& Golden, J. (1999). The route between rapid naming and reading progress. Scientific Studies of Reading, 3, 31-53.

Brass, M., \& von Cramon, D. Y. (2002). The role of the frontal cortex in task preparation. Cerebral Cortex, 12, 908-914.

Brass, M., \& von Cramon, D. Y. (2004). Selection for cognitive control: A functional magnetic resonance imaging study on the selection of task-relevant information. Journal of Neuroscience, 24, 8847-8852.

Braver, T. S., Reynolds, J. R., \& Donaldson, D. I. (2003). Neural mechanisms of transient and sustained cognitive control during task switching. Neuron, 39, 713-726.

Bunge, S. A., Kahn, I., Wallis, J. D., Miller, E. K., \& Wagner, A. D. (2003). Neural circuits subserving the retrieval and maintenance of abstract rules. Journal of Neurophysiology, 90, 3419-3428.

Calabria, M., Hernández, M., Branzi, F. M., \& Costa, A. (2012). Qualitative differences between bilingual language control and executive control: Evidence from task-switching. Frontiers in Psychology, 2, 399.

Costa, A., Hernández, M., \& Sebastián-Gallés, N. (2008). Bilingualism aids conflict resolution: Evidence from the ANT task. Cognition, 106, 59-86.

Costa, A., \& Santesteban, M. (2004). Lexical access in bilingual speech production: Evidence from language switching in highly proficient bilinguals and L2 learners. Journal of Memory and Language, 50, 491-511.

Costa, A., Santesteban, M., \& Ivanova, I. (2006). How do highly proficient bilinguals control their lexicalization process? Inhibitory and language-specific selection mechanisms are both functional. Journal of Experimental Psychology: Learning, Memory, and Cognition, 32, 1057.

Crinion, J., Turner, R., Grogan, A., Hanakawa, T., Noppeney, U., Devlin, J. T., et al. (2006). Language control in the bilingual brain. Science, 312, 1537-1540.

Crone, E. A., Wendelken, C., Donohue, S. E., \& Bunge, S. A. (2006). Neural evidence for dissociable components of task-switching. Cerebral Cortex, 16, 475-486.

Davis, C. J. (2005). N-Watch: A program for deriving neighborhood size and other psycholinguistic statistics. Behavior Research Methods, 37, 65-70.

De Baene, W., Albers, A. M., \& Brass, M. (2012). The what and how components of cognitive control. Neuroimage, 63, 203-211.

De Baene, W., \& Brass, M. (2011). Cue-switch effects do not rely on the same neural systems as task-switch effects. Cognitive, Affective, \& Behavioral Neuroscience, 11, 600-607. 
Denckla, M. B., \& Rudel, R. (1974). Rapid "automatized" naming of pictured objects, colors, letters and numbers by normal children. Cortex, 10, 186-202.

Dove, A., Pollmann, S., Schubert, T., Wiggins, C. J., \& Yves von Cramon, D. (2000). Prefrontal cortex activation in task switching: An event-related fMRI study. Cognitive Brain Research, 9, 103-109.

Dreher, J. C., \& Berman, K. F. (2002). Fractionating the neural substrate of cognitive control processes. Proceedings of the National Academy of Sciences, U.S.A., 99, 14595-14600.

Dreher, J. C., Koechlin, E., Ali, S. O., \& Grafman, J. (2002). The roles of timing and task order during task switching. Neuroimage, 17, 95-109.

Duñabeitia, J. A., Casaponsa, A., Dimitropoulou, M., Martí, A., Larraza, S., \& Carreiras, M. (in preparation). BaSp: A BasqueSpanish database of translation equivalents.

Duñabeitia, J. A., Hernández, J. A., Antón, E., Macizo, P., Estévez, A., Fuentes, L. J., et al. (2014). The inhibitory advantage in bilingual children revisited: Myth or reality? Experimental Psychology, 61, 234.

Friedman, N. P., Miyake, A., Corley, R. P., Young, S. E., DeFries, J. C., \& Hewitt, J. K. (2006). Not all executive functions are related to intelligence. Psychological Science, 17, 172-179.

Garbin, G., Costa, A., Sanjuan, A., Forn, C., Rodriguez-Pujadas, A., Ventura, N., et al. (2011). Neural bases of language switching in high and early proficient bilinguals. Brain and Language, 119, 129-135.

Garbin, G., Sanjuan, A., Forn, C., Bustamante, J. C., RodríguezPujadas, A., Belloch, V., et al. (2010). Bridging language and attention: Brain basis of the impact of bilingualism on cognitive control. Neuroimage, 53, 1272-1278.

Gollan, T. H., \& Ferreira, V. S. (2009). Should I stay or should I switch? A cost-benefit analysis of voluntary language switching in young and aging bilinguals. Journal of Experimental Psychology: Learning, Memory, and Cognition, 35, 640 .

Gottlieb, J. (2007). From thought to action: The parietal cortex as a bridge between perception, action, and cognition. Neuron, 53, 9-16.

Green, D. W. (1998). Mental control of the bilingual lexicosemantic system. Bilingualism: Language and Cognition, 1, $67-81$.

Guo, T., Liu, H., Misra, M., \& Kroll, J. F. (2011). Local and global inhibition in bilingual word production: fMRI evidence from Chinese-English bilinguals. Neuroimage, 56, 2300-2309.

Hartstra, E., Kühn, S., Verguts, T., \& Brass, M. (2011). The implementation of verbal instructions: An fMRI study. Human Brain Mapping, 32, 1811-1824.

Hedden, T., \& Gabrieli, J. D. (2010). Shared and selective neural correlates of inhibition, facilitation, and shifting processes during executive control. Neuroimage, 51, 421-431.

Hernandez, A. E. (2009). Language switching in the bilingual brain: What's next? Brain and Language, 109, 133-140.

Hernandez, A. E., Dapretto, M., Mazziotta, J., \& Bookheimer, S. (2001). Language switching and language representation in Spanish-English bilinguals: An fMRI study. Neuroimage, 14, 510-520.

Hernandez, A. E., Martinez, A., \& Kohnert, K. (2000). In search of the language switch: An fMRI study of picture naming in Spanish-English bilinguals. Brain and Language, 73, 421-431.

Hernández, M., Martin, C. D., Barceló, F., \& Costa, A. (2013). Where is the bilingual advantage in task-switching? Journal of Memory and Language, 69, 257-276.

Hester, R., D’Esposito, M., Cole, M. W., \& Garavan, H. (2007). Neural mechanisms for response selection: Comparing selection of responses and items from working memory. Neuroimage, 34, 446-454.
Hikosaka, O., \& Isoda, M. (2010). Switching from automatic to controlled behavior: Cortico-basal ganglia mechanisms.

Trends in Cognitive Sciences, 14, 154-161.

Hillis, A. E., Work, M., Barker, P. B., Jacobs, M. A., Breese, E. L., \& Maurer, K. (2004). Re-examining the brain regions crucial for orchestrating speech articulation. Brain, 127, 1479-1487.

Hoshi, E., \& Tanji, J. (2007). Distinctions between dorsal and ventral premotor areas: Anatomical connectivity and functional properties. Current Opinion in Neurobiology, 17, 234-242

Hyafil, A., Summerfield, C., \& Koechlin, E. (2009). Two mechanisms for task switching in the prefrontal cortex. Journal of Neuroscience, 29, 5135-5142.

Isoda, M., \& Hikosaka, O. (2007). Switching from automatic to controlled action by monkey medial frontal cortex. Nature Neuroscience, 10, 240-248.

Jackson, G. M., Swainson, R., Cunnington, R., \& Jackson, S. R. (2001). ERP correlates of executive control during repeated language switching. Bilingualism: Language and Cognition, 4, $169-178$

Kaplan, E., Goodglass, H., \& Weintraub, S. (1983). The Boston Naming Test. Philadelphia, PA: Lea \& Febiger.

Kim, C., Cilles, S. E., Johnson, N. F., \& Gold, B. T. (2012). Domain general and domain preferential brain regions associated with different types of task switching: A metaanalysis. Human Brain Mapping, 33, 130-142.

Kimberg, D. Y., Aguirre, G. K., \& D'Esposito, M. (2000). Modulation of task-related neural activity in task-switching: An fMRI study. Cognitive Brain Research, 10, 189-196.

Luk, G., Anderson, J. A., Craik, F. I., Grady, C., \& Bialystok, E. (2010). Distinct neural correlates for two types of inhibition in bilinguals: Response inhibition versus interference suppression. Brain and Cognition, 74, 347-357.

Luk, G., Green, D. W., Abutalebi, J., \& Grady, C. (2012). Cognitive control for language switching in bilinguals: A quantitative meta-analysis of functional neuroimaging studies. Language and Cognitive Processes, 27, 1479-1488.

Ma, H., Hu, J., Xi, J., Shen, W., Ge, J., Geng, F., et al. (2014). Bilingual cognitive control in language switching: An fMRI study of English-Chinese late bilinguals. PLoS One, 9, e106468.

MacDonald, A. W., Cohen, J. D., Stenger, V. A., \& Carter, C. S. (2000). Dissociating the role of the dorsolateral prefrontal and anterior cingulate cortex in cognitive control. Science, 288, 1835-1838

Mayka, M. A., Corcos, D. M., Leurgans, S. E., \& Vaillancourt, D. E. (2006). Three-dimensional locations and boundaries of motor and premotor cortices as defined by functional brain imaging: A meta-analysis. Neuroimage, 31, 1453-1474.

Meuter, R. F., \& Allport, A. (1999). Bilingual language switching in naming: Asymmetrical costs of language selection. Journal of Memory and Language, 40, 25-40.

Mevorach, C., Humphreys, G. W., \& Shalev, L. (2006). Opposite biases in salience-based selection for the left and right posterior parietal cortex. Nature Neuroscience, 9, 740-742.

Miyake, A., Friedman, N. P., Emerson, M. J., Witzki, A. H., Howerter, A., \& Wager, T. D. (2000). The unity and diversity of executive functions and their contributions to complex "frontal lobe" tasks: A latent variable analysis. Cognitive Psychology, 41, 49-100.

Murtha, S., Chertkow, H., Beauregard, M., \& Evans, A. (1999). The neural substrate of picture naming. Journal of Cognitive Neuroscience, 11, 399-423.

O'Shea, J., Johansen-Berg, H., Trief, D., Göbel, S., \& Rushworth, M. F. (2007). Functionally specific reorganization in human premotor cortex. Neuron, 54, 479-490.

Paap, K. R., \& Greenberg, Z. I. (2013). There is no coherent evidence for a bilingual advantage in executive processing. Cognitive Psychology, 66, 232-258. 
Peelen, M. V., \& Downing, P. E. (2005). Within-subject reproducibility of category-specific visual activation with functional MRI. Human Brain Mapping, 25, 402-408.

Peelen, M. V., Wiggett, A. J., \& Downing, P. E. (2006). Patterns of fMRI activity dissociate overlapping functional brain areas that respond to biological motion. Neuron, 49, 815-822.

Pouget, A., Deneve, S., \& Duhamel, J. R. (2002). A computational perspective on the neural basis of multisensory spatial representations. Nature Reviews Neuroscience, 3, 741-747.

Prior, A., \& Gollan, T. H. (2011). Good language-switchers are good task-switchers: Evidence from Spanish-English and Mandarin-English bilinguals. Journal of the International Neuropsychological Society, 17, 682-691.

Prior, A., \& MacWhinney, B. (2010). A bilingual advantage in task switching. Bilingualism: Language and Cognition, 13, 253-262.

Ridderinkhof, K. R., Ullsperger, M., Crone, E. A., \& Nieuwenhuis, S. (2004). The role of the medial frontal cortex in cognitive control. Science, 306, 443-447.

Rossion, B., \& Pourtois, G. (2004). Revisiting Snodgrass and Vanderwart's object pictorial set: The role of surface detail in basic-level object recognition. Perception, 33, 217-236.

Ruge, H., Brass, M., Koch, I., Rubin, O., Meiran, N., \& Cramon, D. (2005). Advance preparation and stimulus-induced interference in cued task switching: Further insights from BOLD fMRI. Neuropsychologia, 43, 340-355.

Rushworth, M. F., Paus, T., \& Sipila, P. K. (2001). Attention systems and the organization of the human parietal cortex. Journal of Neuroscience, 21, 5262-5271.

Rushworth, M. F. S., Hadland, K. A., Paus, T., \& Sipila, P. K. (2002). Role of the human medial frontal cortex in task switching: A combined fMRI and TMS study. Journal of Neurophysiology, 87, 2577-2592.

Ruthruff, E., Remington, R. W., \& Johnston, J. C. (2001). Switching between simple cognitive tasks: The interaction of top-down and bottom-up factors. Journal of Experimental Psychology: Human Perception and Performance, 27, 1404

Serrien, D. J., Ivry, R. B., \& Swinnen, S. P. (2007). The missing link between action and cognition. Progress in Neurobiology, 82, 95-107.
Shi, Y., Zhou, X., Müller, H. J., \& Schubert, T. (2010). The neural implementation of task rule activation in the task-cuing paradigm: An event-related fMRI study. Neuroimage, 51, 1253-1264.

Sohn, M. H., Ursu, S., Anderson, J. R., Stenger, V. A., \& Carter, C. S. (2000). The role of prefrontal cortex and posterior parietal cortex in task switching. Proceedings of the National Academy of Sciences, 97, 13448-13453.

Torgesen, J. K., Wagner, R. K., Rashotte, C. A., Burgess, S., \& Hecht, S. (1997). Contributions of phonological awareness and rapid automatic naming ability to the growth of word-reading skills in second- to fifth-grade children. Scientific Studies of Reading, 1, 161-185.

Van Heuven, W. J., Schriefers, H., Dijkstra, T., \& Hagoort, P. (2008). Language conflict in the bilingual brain. Cerebral Cortex, 18, 2706-2716.

Verhoef, K., Roelofs, A., \& Chwilla, D. J. (2009). Role of inhibition in language switching: Evidence from event-related brain potentials in overt picture naming. Cognition, 110, 84-99.

Verreyt, N., Woumans, E., Vandelanotte, D., Szmalec, A., \& Duyck, W. (2015). The influence of language switching experience on the bilingual executive control advantage. Language and Cognition. doi: 10.1017/S1366728914000352.

Wagner, R. K., \& Torgesen, J. K. (1987). The nature of phonological processing and its causal role in the acquisition of reading skills. Psychological Bulletin, 101, 192.

Wang, Y., Kuhl, P. K., Chen, C., \& Dong, Q. (2009). Sustained and transient language control in the bilingual brain. Neuroimage, 47, 414-422.

Wang, Y., Xue, G., Chen, C., Xue, F., \& Dong, Q. (2007). Neural bases of asymmetric language switching in second-language learners: An ER-fMRI study. Neuroimage, 35, 862-870.

Weissberger, G. H., Wierenga, C. E., Bondi, M. W., \& Gollan, T. H. (2012). Partially overlapping mechanisms of language and task control in young and older bilinguals. Psychology and Aging, 27, 959.

Woolgar, A., Thompson, R., Bor, D., \& Duncan, J. (2011). Multi-voxel coding of stimuli, rules, and responses in human frontoparietal cortex. Neuroimage, 56, 744-752.

Yeung, N., Nystrom, L. E., Aronson, J. A., \& Cohen, J. D. (2006). Between-task competition and cognitive control in task switching. Journal of Neuroscience, 26, 1429-1438. 